\title{
Compression of map images for real-time applications
}

\author{
Pasi Fränti*, Eugene Ageenko, Pavel Kopylov, Sami Gröhn, Florian Berger \\ Department of Computer Science, University of Joensuu, P.O. Box 111, FIN-80101 Joensuu, Finland
}

Received 4 June 2001; received in revised form 28 April 2004; accepted 19 May 2004

\begin{abstract}
Digital maps can be stored and distributed electronically using compressed raster image formats. We introduce a storage system for the map images that supports compact storage size, decompression of partial image, and smooth transitions between various scales. The main objective of the proposed storage system is to provide map images for real-time applications that use portable devices with low memory and computing resources. Compact storage size is achieved by dividing the maps into binary layers, which are compressed using context-based statistical modeling and arithmetic coding. Partial image decompression is supported by tiling the image into blocks and implementing direct access to the compressed blocks. In this paper, we give overview of the system architecture, describe the compression technique, and discuss implementation aspects. Experimental results are given both in terms of compression ratios and image retrieval timings.

(c) 2004 Elsevier B.V. All rights reserved.
\end{abstract}

Keywords: Image compression; Map images; Real-time applications; Personal navigation; Spatial access

\section{Introduction}

Real-time cartography imaging application provides user with the view of geographic map for the area surrounding the user's location [1,2]. The system may use global positioning service (GPS) [3] or mobile positioning service (MPS) [4] for obtaining the coordinates of the current location. The location can be updated in real-time (about once or twice in every second). The system must also support real-time panning (spatial movement) and zooming (change of resolution) on the map. By panning, we mean scrolling the map; and by zooming, we mean the change of the view on the display in a closer or wider perspective.

Digital maps are usually obtained from spatial databases $[5,6]$ where the maps are stored in vector formats. The visual outlook of maps representing the same region varies depending on the type of the map (topographic or road map), and on the desired scale (local and regional maps). Individual map images are reproduced for each scale separately and stored as separate raster images augmented with the location information of the map. A typical map image needs only a few color tones but high spatial

\footnotetext{
* Corresponding author. Tel.: +358-13-2515271; fax: +358-132513290.

E-mail address: franti@cs.joensuu.fi (P. Fränti).
}

resolution for representing the details such as roads, infrastructure and names of the places.

In on-line map imaging applications, the images are usually stored in an inefficient, uncompressed raster form. The storage size of a map image is huge. For example, electronic library of Finnish road maps of the resolution 1:250,000 takes an entire CD (over $600 \mathrm{Mb}$ ) in uncompressed form [7]. In comparison, the portable viewing device, such as pocket computers, have typically about $64 \mathrm{Mb}$ of the storage space, which can be expanded at present by about $256 \mathrm{Mb}$ through using compact flash memory cards [8]. The storage requirements of the maps can therefore be a bottleneck, especially in the case of portable devices, in which the maps share the limited memory resources with the operating system, application and other data.

A better approach is to provide the user with the images in compressed form $[9,10]$. For example, an uncompressed black-and-white topographic image of $5000 \times 5000$ pixels takes about $3 \mathrm{Mb}$ in uncompressed form. The latest compression standards [11], however, can compress typical map images by a factor of about $20: 1$, which corresponds to the file size of $150 \mathrm{~kb}$. A drawback of the existing compression techniques is that the entire image must be decompressed in memory before the image can be viewed. 


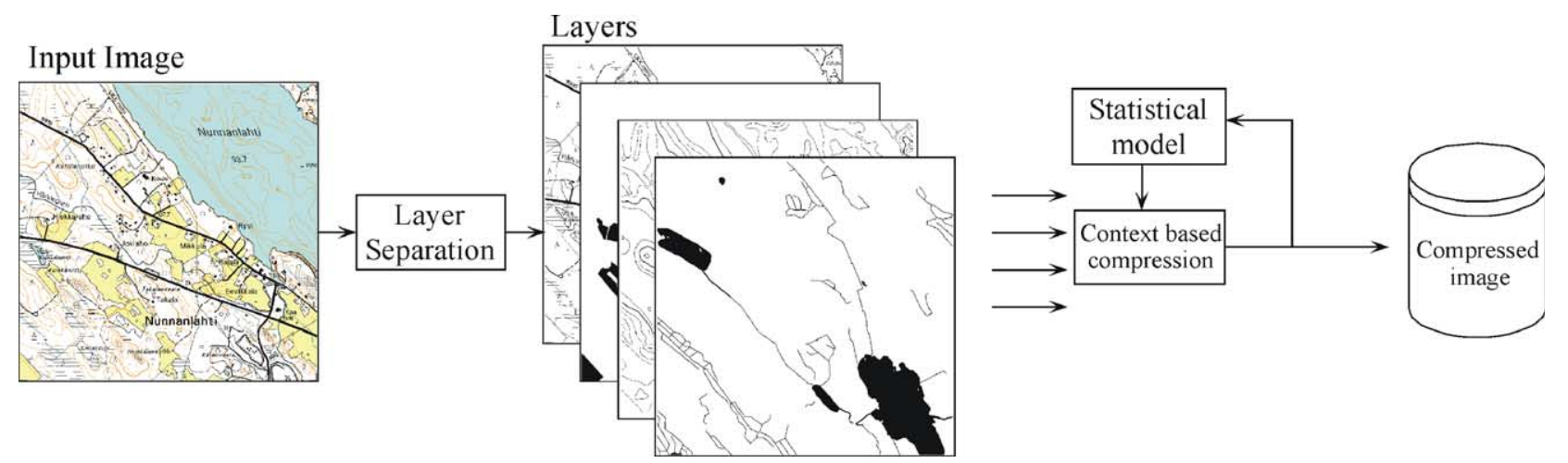

Fig. 1. Outline of the map image compression system.

This can be a problem if the device does not have sufficient computing resources for real-time image decompression.

In this paper, we propose map image storage system (further denoted as MISS), in which we present reasonable solutions both to the storage problem and to the real-time requirements of the system. The MISS images are composed of semantic binary layers, which are compressed using a context-based statistical modeling and arithmetic coding as shown in Fig. 1. The method is basically the same as in the latest international compression standards, Joint Bi-level Image Group (JBIG) and JBIG2 [12-14] with a few differences described later.

To meet the real-time requirements, we provide direct access to the compressed image file. Our approach is to divide the image into $b \times b$ non-overlapping rectangular blocks, which are compressed separately. The compressed blocks are stored in the same file, and an index table is stored in the header of the file to locate the starting points of the code blocks. In this way, direct access can be provided with the accuracy of the block size. The block size is a compromise between compression efficiency and the decoding speed. The JBIG2 file structure supports this kind of file organization, where the image is composed of several segments with direct access.

The rest of the paper is organized as follows. The proposed MISS is introduced in Section 2. Multi-scale representation of the map is first discussed in Section 2.1, and the compression method briefly recalled in Section 2.2. The decomposition of the image into binary layers is studied in Section 2.3. The image tiling into blocks for supporting efficient panning in studied in Section 2.4. The file structure and the proposed system architecture are summarized in Section 2.5. Experimental results are given in Section 3 to demonstrate the compression performance and the decoding efficiency of the system in real-time environment. Conclusions are drawn in Section 4.

\section{Map image storage system}

Digital maps are usually stored as vector graphics in a database for retrieving the data using the spatial location as the search key. Vector representation is convenient for zooming as the maps can be displayed in any resolution defined by the user. Panning of the map can be performed by retrieving the elements needed for updating the changes in the view. The use of database, however, can be impractical in mobile environment, as the devices may not have enough resources to store the complete map database and the database engine.

The storage problem could be solved by generating spatial views (mapsheets) from the database and store the maps in a vector format. The storage size can be reduced further by compressing the vector maps (by a factor of about $2: 1)$, or by simplifying the vector representation. This approach, however, does not support real-time panning as separate data structures must be built for this purpose.

The biggest problem of the vector format is that maps are not always available for the user in vector format. Moreover, the maps are stored in various formats and incompatibility between different systems can restrict the use of the maps. To sum up, vector format is a good approach if the user has sufficient hardware and software resources, and if the maps are widely available in a compatible vector format. Otherwise, raster image format is the only choice.

We introduce next a MISS based on compressed raster format. The system support the following properties of the maps:

1. Compact storage size

2. Multi-scale representation (zooming)

3. Fast scrolling ability (panning).

The idea is that the maps are stored in server-side database. Spatial views are generated for the client-side application using compressed raster image format organized so that it supports the zooming and panning requirements. In this way, raster format is suitable in applications, where the maps are needed for viewing purposes only.

Furthermore, the system does not depend on any database or vector format as digitized raster maps can be easily generated and reproduced from any source format, including paper maps. The conversion of the maps 

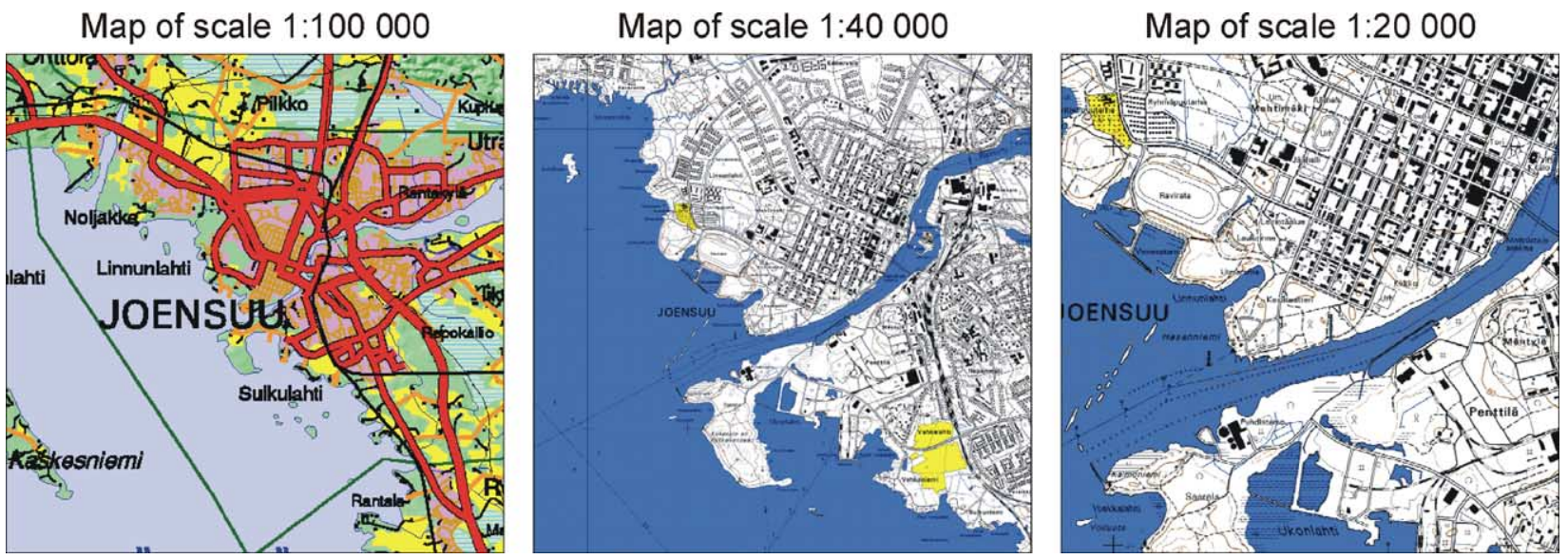

Fig. 2. Example of a map shown in three different scales. The highest and smallest scales have different representation of the content, but the intermediate scale $(1: 40,000)$ has been generated from the map of lowest scale $(1: 20,000)$.

is therefore not a big problem. Another advantage of the system is that it requires only a modest memory and computing resources in order to be operational in real-time environment.

\subsection{Multi-scale representation}

The visual outlook of the maps varies depending on the scale. It is therefore not convenient to use multi-resolution image representation. Instead, several different map images should be reproduced for each desired scale. In addition to this, intermediate scales can also be provided by zooming the raster image. For example, Fig. 2 includes two different scales of a map $(1: 20,000$ and $1: 100,000)$ of the same location. The intermediate scale 1:40,000 has been generated from the detailed map $(1: 20,000)$ in order to provide the user with smoother zooming. The image has the same level of details but the size and quality of the features suffer because of the change in resolution.

The intermediate scale images can be generated beforehand, and stored in the library. Another solution is to perform real-time zooming in the client application. The organization of the data is illustrated in Fig. 3. In this example, the large rectangles represent the map images of four different scales. The size of the rectangles corresponds to the size of the individual images in pixels. The images have usually the same size when printed on paper or shown on display. The thin grid lines drawn across the rectangles correspond to the spatial territories shown in the maps that have same size in reality but different resolution.

\subsection{Image compression}

A compressed raster image format provides a reasonable solution in the form of compact storage size and compatible map format. Typical map images have high spatial resolution for representing fine details such as text and graphics objects but not so much color tones as photographic images. The most suitable compression methods can thus be found among the lossless graphics compression methods such as Graphics Interchange Format (GIF) and Portable Network Graphics (PNG) [15,16]. It is also possible to divide the maps into separate color layers and to apply the lossless binary image compression standards such as ITU-T Group 4 [9], or the current standard JBIG2 [13,14]. However, lossy compression methods, such as the JPEG [17] do not apply well for map images.

We take the JBIG1 and JBIG2 as the starting point of our MISS. They use context-based statistical modeling and arithmetic coding in the manner as originally proposed in Ref. [18]. The image is processed pixel-by-pixel in raster-scan order. The probability of each pixel is estimated on the basis of previous occurrences in similar context. The context is defined as the combination of already processed neighboring pixels defined by a template. Each context is assigned with its own statistical model that is adaptively updated during the compression process. Decompression is synchronous process with compression.

Here, we apply the JBIG2 file format but use only the generic mode, which is basically the same as JBIG1. JBIG2 also segments the image into regions of different types,

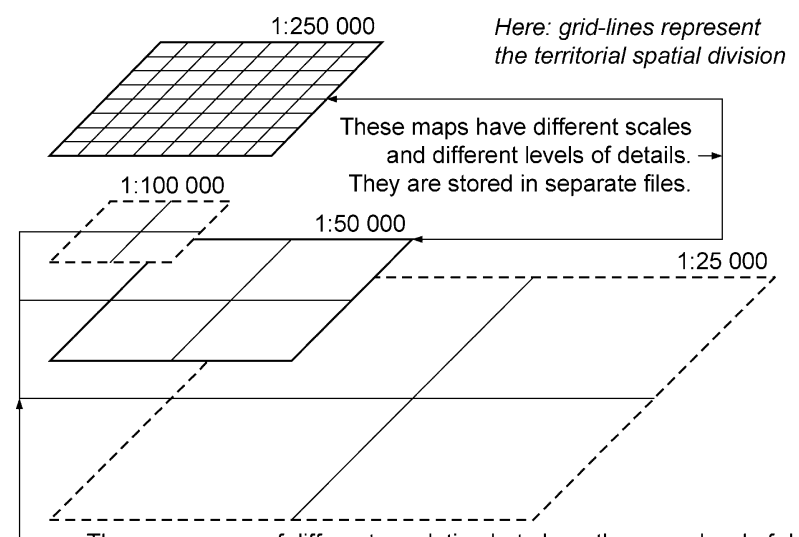

These maps are of different resolution but share the same level of detail. They are generated from 1:100 000 map on the fly.

Fig. 3. Representation of the maps as separate map images (1:250,000 and $1: 50,000)$, and as intermediate scales that are obtained by zooming. 


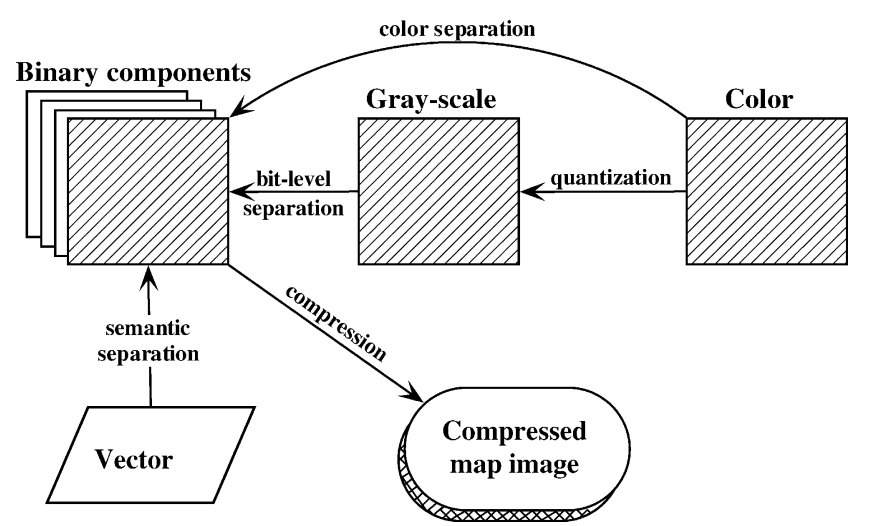

Fig. 4. Conversion diagram for using layer separation and binary image compression methods.

in particular, textual, halftone and generic (other), and utilize the repetitive nature of the textual and halftone images. However, the encoding of the data other than text or halftones remains similar to JBIG with the difference that a newer version of the arithmetic coder (MQ-coder) is used. The pre-ancestor, $Q$-coder, has similar working principles [19]. For more details about the application of JBIG2 file structure (see Ref. [20]).

Better compression could be achieved by using multi-layer context tree modeling as recently proposed in Ref. [21] but at the cost of much longer encoding time.
However, in this work, we content ourselves with the standard compression schemes as we emphasize the simplicity and compatibility of the proposed map imaging system.

\subsection{Decomposition to binary layers}

In order to utilize the context-based compression, the map must be divided into binary layers. Each layer is then compressed separately, and the compressed layers are stored into the same file. There are three ways to perform the decomposition as illustrated in Fig. 4:

1. Semantic decomposition

2. Color separation

3. Bit-level separation.

Semantic decomposition is possible if the maps are obtained from a map database in vector format. The map is output into a set of binary layers each containing different semantic meaning. We consider maps that consist of five layers: basic (topographic data and contours), elevation lines, fields, water and property (administrative borders, see Fig. 5). The user application can reproduce the map by plotting each layer by its own color overlapping each other in a given order. Color information can be added into the file.

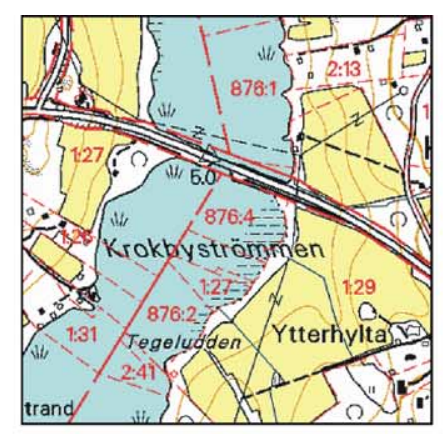

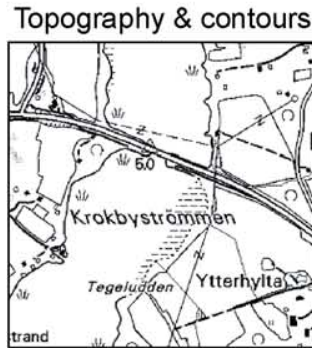

Color 1
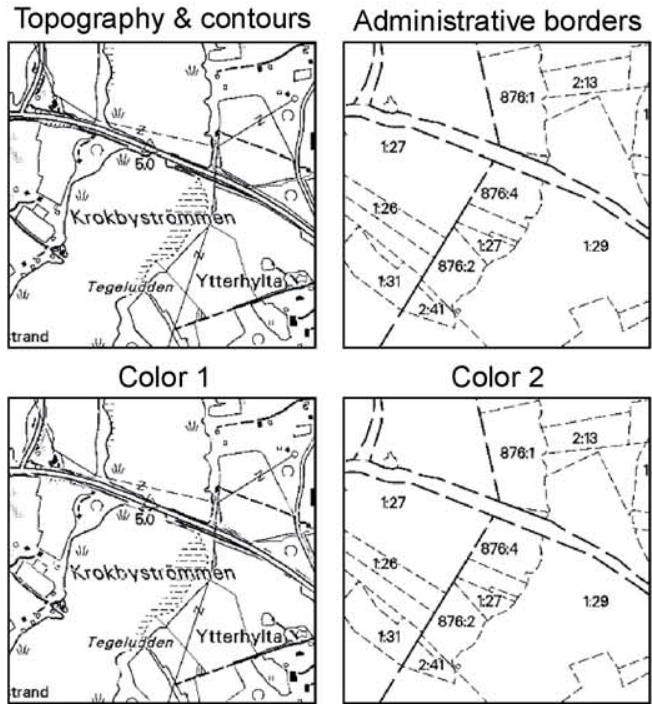

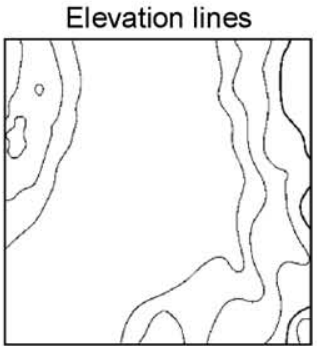

Color 3

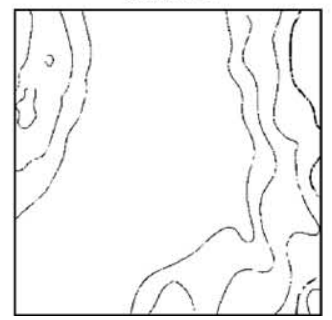

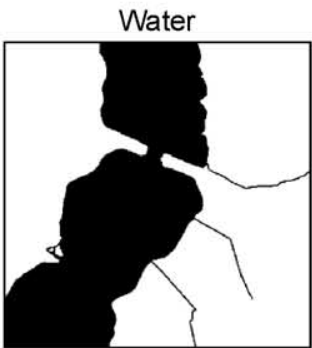

Color 4

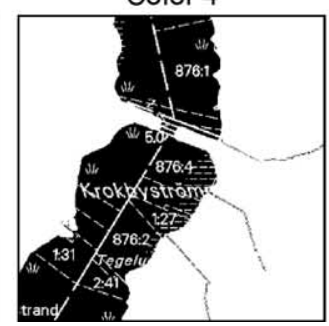

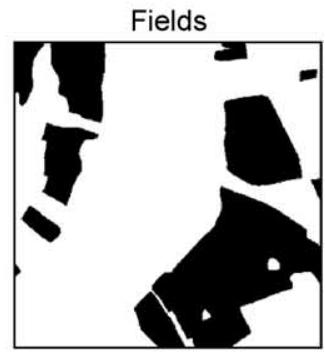

Color 5

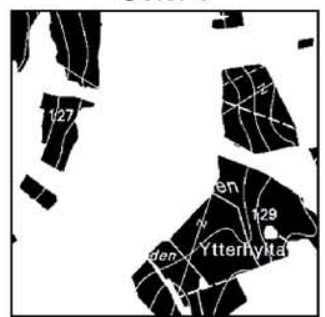

Fig. 5. Sample fragment from a color map image (above), and its decomposition into binary layers by semantic separation (middle), color separation (below). 


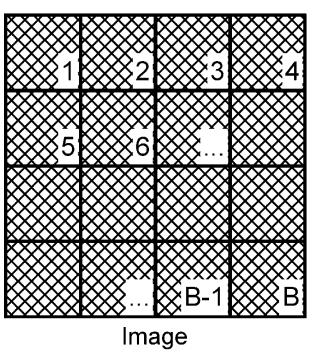

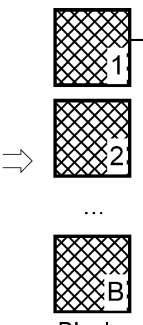

Blocks

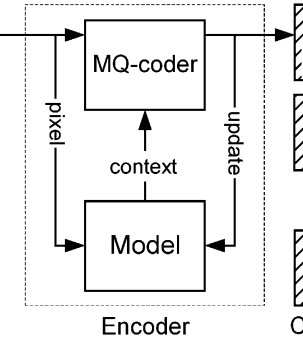

Encoder

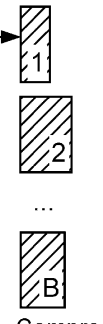

bompross

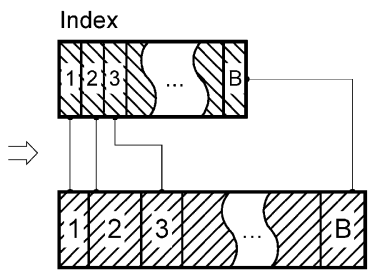

Sequential buffer

Fig. 6. Diagram of the block decomposition.

The image can be reconstructed as a gray-scale image, or using any color set given by the user application. The benefits of the semantic separation are better compression performance, and that the layers to be shown can be selected at the time of viewing.

The second approach, color separation, can be used when we have only raster color image as the original map, and it contains only a limited number of colors [22]. The image is divided into binary layers so that each layer represents one color in the original image. The drawback of the color separation is that information of the original semantic separation cannot be recovered. Furthermore, the color separation can create artifacts into the binary layers (see Fig. 5). For example, overlapping text elements break the continuation of the fields and lakes. This does not decrease the quality of the image but it increases the complexity of the image, and thus, the compressed file size.

The third approach, bit-level separation, must be applied when we have the original map only as a raster image, and the number of different colors is too high for efficient color separation. For example, the image might have been digitized from a paper copy and stored using lossy compression method, such as JPEG [17]. In the bit-level separation, the number of colors are first reduced by quantizing the image into a limited-color representation of a 256 colors or gray scales. The resulting pixel values are then separated into bit planes using gray coding [23], and the image is represented as a sequence of binary images.

\subsection{Block decomposition for direct access}

The binary layers are divided into $b \times b$ non-overlapping rectangular blocks before the compression, and each block is compressed separately from others as proposed in Ref. [24]. The compressed blocks are stored in the same file, and an index table is stored in the header of the file to locate the starting points of the code blocks (see Fig. 6). When the compressed image is accessed, a block index table is constructed. This provides direct access to the compressed image file, and therefore, enables efficient decompression of a particular image fragment.

The block decomposition has the effect that there are fewer pixels to be coded in the same run. It means that the model has less time to adapt to the statistics of the image.
Another problem is the compression inefficiency near block boundaries. This is because the pixels located outside the block cannot be used in the context template. Previous studies indicate that the compression inefficiency remains tolerable if the block size is $256 \times 256$ pixels or higher [24].

Somewhat better compression performance can be obtained using the following two modifications. First idea is to apply a forward-adaptive variant of the statistical modeling based on the ideas presented in Ref. [26]. The forward-adaptive variant uses a pre-calculated initial model, which is constructed using the statistics collected from the entire image layer. This requires an additional pass over the image but it does not affect the speed of the decompression. The second idea is to use variable-size context modeling technique as described in Ref. [25]. This technique reduces the size of the model, and it allows using larger context templates. These modifications can provide about $20 \%$ improvement in the compression performance.

\subsection{Compression phase}

The compression of a single map image is performed using the following steps:

1. Layer decomposition

2. Block decomposition

3. Compression.

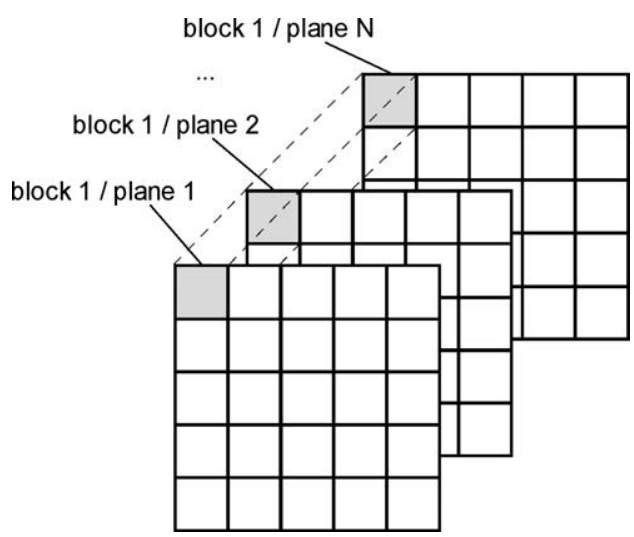

Fig. 7. Image decomposition diagram. 

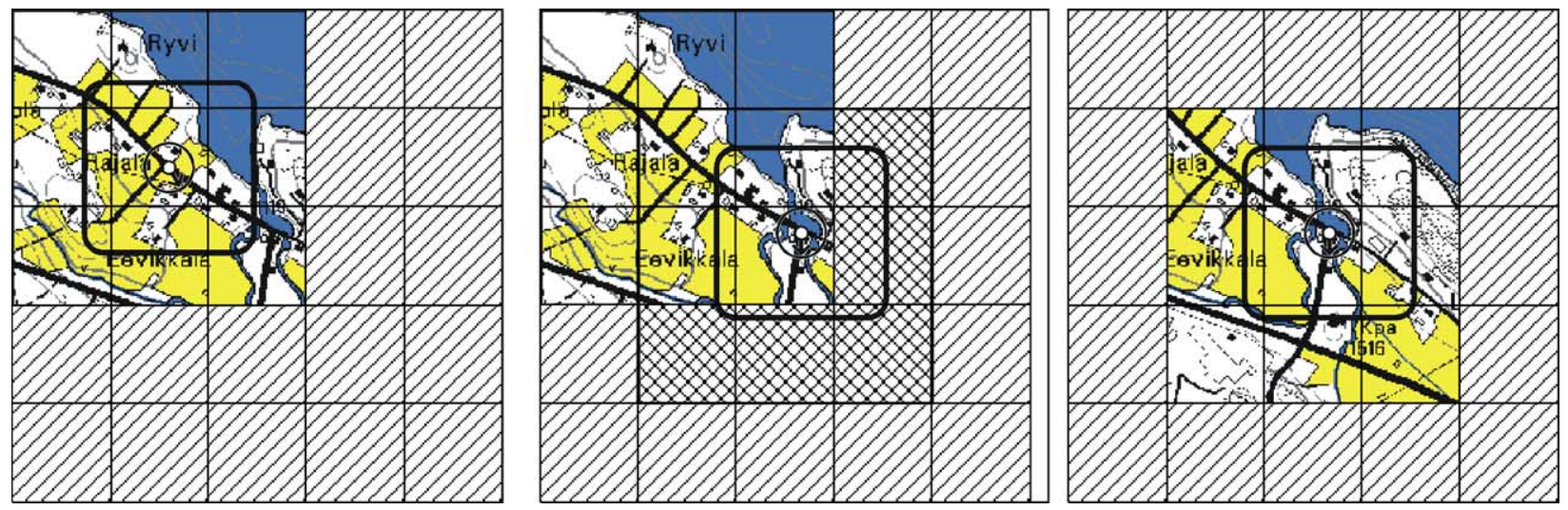

Fig. 8. Image decoding in real-time system. Nine blocks are first decompressed and stored in the cache (left). Change of location is then registered (middle), and new image blocks are then decompressed and the view updated (right).

In the first step, the image is decomposed into the binary layers (unless the semantic decomposition already exists) The color space is enumerated and the number of required bit planes are generated. The resulting color palette is stored in the compressed file. If the palette is not stored, the image will be reconstructed as a gray scale.

In the second step, the layers are partitioned into blocks. If the forward-adaptive modeling variant is applied, non-empty blocks of the entire layer are analyzed and the statistical model is built and stored in the compressed file. The emptiness of a block is determined by checking whether all pixel values in the block are of the default color value.

In the third step, the series of the bit planes are compressed and ordered highest to lowest (see Fig. 7). Each non-empty block is compressed using context-based modeling and the MQ-coding algorithm as described in Section 2.2, which is basically the same as the compression of separate generic regions in JBIG2. The context is determined by a fixed-size template but we also permit the use of custom contexttemplates as described in Ref. [27]. The MQ-coder is reinitialized every time the compression of a new block starts. The initialization resets the model to the blank model (default), or to the optimized initial model (optional).

\subsection{Use in the client device}

A typical use scenario of the MISS is to show the area surrounding the object whose position is tracked The scale to be used can be set by the user or it can be automatically determined on the basis of the speed, or other parameters defined in the application. The maps for the particular region are stored in the client's viewing device (flash memory, hard disk, CD), or the images can be located in a remote server and accessed via communication network (Internet, GSM, GRPS).

The system uses the following steps to show the current view on the map. First, the file possessing the map of the desired location is accessed and its header part is retrieved in memory. From the header, the type and structure of the image are determined, and the block index table is built. The table indicates the size and location of each block in the compressed file. All supplementary data required for image decoding (such as initial model and possible context tree) are also retrieved and kept in memory until this particular map image is no longer used.

Next, depending on the requested location, the system calculates the map image fragment needed to be displayed. The blocks covering this fragment are retrieved

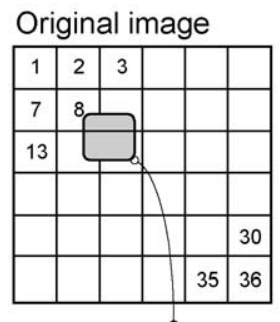

Cache

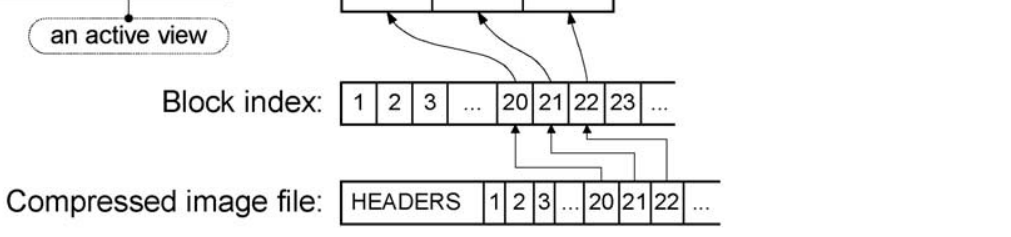

Fig. 9. Illustration of the cache operation. 


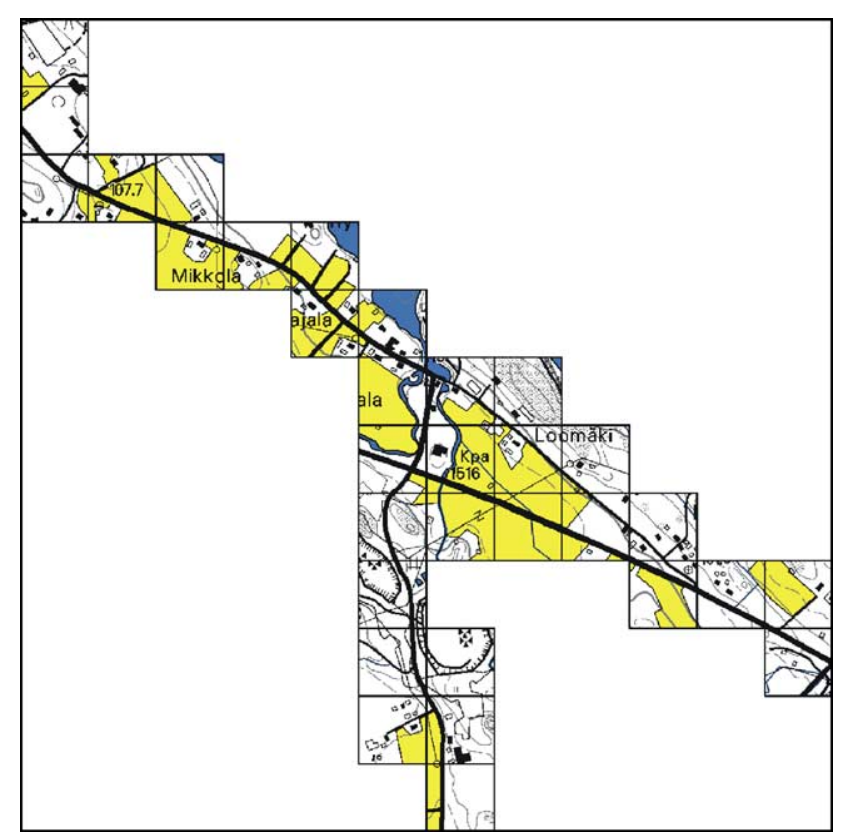

Fig. 10. Dynamic map handling by composing map sheet from individual map segments.

and decoded. If the map image is accessed remotely, the retrieved blocks are also stored (in compressed form) in the local storage space for further use. When the position of the object changes, the view is updated by decoding new image blocks in the direction of movement (see Fig. 8).

To speed-up the access to the image, the system may use cache for temporary storage of the decompressed image blocks (see Fig. 9). The size of the cache can be fixed, or it can be determined by the amount of free memory. The performance may be further improved by exploiting an idle time for decoding neighboring blocks further in the direction of movement, before the blocks are actually requested. In practice, it is convenient to buffer the data according to the block boundaries.

On the basis of the proposed MISS, a fully dynamic map handing system can be built as proposed in Ref. [28]. When there is no map of the current location the client creates a blank map image and then begins to request relevant blocks from the servers as shown in Fig. 10. The image is gradually built up by the addition of new blocks. When the mobile device memory is full, less relevant maps are removed. This requires that the concept of relevance be well defined as to minimize the cost of map transmission. The addition of new data must be implemented at the block-level but removal may occur, for the sake of simplicity, only at the file level.

It is expected that dynamic map handling can be integrated with a mobile phone or GPS-based device so that the user pays for map access just once, when buying the device. He will have no further need to worry about map access at all. Scenarios of such applications have been outlined in Ref. [29].

\section{Experimental results}

We study next the compression performance and the retrieval times of the proposed storage system. The following methods are considered in the comparisons:

- MISS

- JBIG2

- TIFF G4

- GIF

- PNG

- RAW

JBIG2 $[13,14]$ is the latest binary image compression standard. We compress the whole image as one region using generic coding with the 10-pixel context template. TIFF G4 [9] refers to the older ITU-T Group 4 fax compression standard; we use the method as included in the Tagged Image File Format (TIFF). The CompuServe Graphics Interchange Format (GIF) [15] is the most widely used format in Internet for palette images. It uses the dictionarybased coding known as LZW [30], which is based on class of Ziv-Lempel methods known as LZ78 [31]. The PNG [16] differs from GIF in that it is based on LZ77 dictionary compression [32] instead of the LZ78.

In MISS, we use the following parameter setup:

- The default 10-pixel context template from JBIG2,

- Forward-adaptive statistical modeling optimized for each layer,

- Block size of $100 \times 100$ pixels,

- Other parameters same as in JBIG2.

\subsection{Test image sets}

We use three image sets representing the following situations:

- Set \#1: layers separation by semantic decomposition,

- Set \#2: layers separation by color separation,

- Set \#3: layers separation by quantization and bit-level separation.

The first two sets contain topographic map images, and the third a set of two road map images. The first two sets originate from the NLS topographic database [33]. The images are of the size $5000 \times 5000$ pixels, and have the original scale 1:20,000. This corresponds to a resolution of two meters per pixel. The topographic map images are composed of five semantic layers representing topography, elevation lines, waterways, fields and administrative boundaries (when available).

The Set \#1 (semantic decomposition) includes the images when separated into the five semantic layers. The Set \#2 (color separation) contains the same set of images but after the following processing. Color image is 

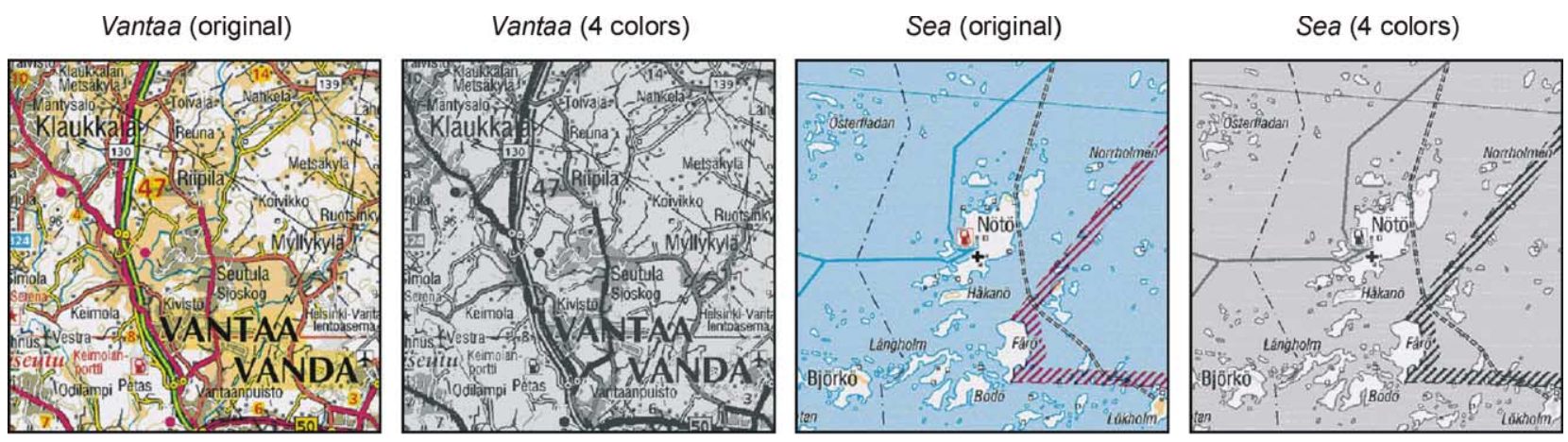

Fig. 11. Sample fragments from the images in the Set \#3.

first constructed from the original semantic layers, and color separation is then performed to estimate the original division. See Fig. 5 for the difference in the bit-plane separation in these two cases. The Set \#3 (bit-level separation) consists of two scanned color map images including scanning noise and a high number of output colors (see Fig. 11). In the experiments, the images have then been converted to 2 bits per pixel gray-scale images corresponding to the number of colors available in a low cost mobile device. The images have been divided into two bit-planes.

\subsection{Compression performance}

The compression results have been summarized in Tables 1-3. The average compression performance of MISS is about 0.20 bits per pixel but the result depends on the complexity of the image. The MISS files take about $5-15 \%$ more space than that the JBIG2 files, on average, but $50-65 \%$ less than the comparative methods (TIFF-G4, GIF, PNG). The MISS, on the other hand, is the only method that supports direct access to the compressed file. The results in Fig. 12 show that most of the bits (about 54\%) originates from the basic information, whereas the water and fields are rather easy to compress. Administrative boundaries were absent in the case of image 3 .

The compression results between the Set \#1 and Set \#2 are not significant. The color separation loses information that originally appears in the image, but at the same time, it adds to the complexity of the images when the layers are compressed separately. The overall effect, however, remains small. We therefore did not consider multi-level context templates, which could be used to handle this kind of inter-layer dependencies. In the case of Set \#2, the GIF and PNG methods were applied to the color images, and not the bit planes. In the case of Set \#1, this is also possible but not desirable, as we did not want to lose the semantic separation of the images.

The images in the Set \#3 are more difficult to compress because of the noise and the level of complexity of the images. The average bit rate for these images is 0.84 . The performance gap between MISS and the comparative methods (TIFF-G4, GIF, PNG) is much smaller than in the case of the Set \#1 and Set \#2 (MISS files take about $25 \%$ less than the PNG files). The results demonstrate the importance of having the image divided into semantic layers, or at least a clean original so that the color separation can be performed.

The effect of the block size is illustrated in Fig. 13. The optimal block size is around $350 \times 350$ to $500 \times 500$ in

Table 1

Compression results (kilobytes) for the set 1 (semantic decomposition)

\begin{tabular}{lcccccr}
\hline & MISS & JBIG2 & TIFF G4 & GIF & PNG & RAW \\
\hline Image 1 & 247 & 197 & 372 & 727 & 602 & 15,259 \\
Image 2 & 1109 & 969 & 2382 & 2866 & 2608 & 15,259 \\
Image 3 & 395 & 327 & 604 & 1142 & 1100 & 15,259 \\
Image 4 & 840 & 759 & 1540 & 2633 & 2534 & 15,259 \\
Total & 2591 & 2252 & 4899 & 7367 & 6845 & 61,035 \\
bpp & 0.21 & 0.18 & 0.40 & 0.60 & 0.56 & 5.00 \\
\hline
\end{tabular}

Table 2

Compression results (kilobytes) for the set 2 (color separation)

\begin{tabular}{lcccccc}
\hline & MISS & JBIG2 & TIFF G4 & GIF $^{\mathrm{a}}$ & PNG $^{\mathrm{a}}$ & RAW \\
\hline Image 1 & 310 & 258 & 466 & 610 & 654 & 15,259 \\
Image 2 & 1269 & 1138 & 2605 & 2642 & 2644 & 15,259 \\
Image 3 & 426 & 360 & 691 & 1028 & 1074 & 15,259 \\
Image 4 & 957 & 875 & 1902 & 2295 & 2384 & 15,259 \\
Total & 2962 & 2631 & 5664 & 6575 & 6757 & 61,035 \\
bpp & 0.24 & 0.22 & 0.46 & 0.54 & 0.55 & 5.00 \\
\hline
\end{tabular}

${ }^{a}$ The GIF and PNG results are obtained by compressing the corresponding color image instead of the binary layers.

Table 3

Compression results (kilobytes) for set 3 (bit-level separation)

\begin{tabular}{lcccccc}
\hline & MISS & JBIG2 & TIFF G4 & GIF $^{\mathrm{a}}$ & PNG $^{\mathrm{a}}$ & RAW \\
\hline Vantaa & 91 & 84 & 123 & 120 & 132 & 313 \\
Sea & 44 & 39 & 72 & 53 & 59 & 313 \\
Total & 135 & 123 & 195 & 173 & 191 & 625 \\
bpp & 0.86 & 0.79 & 1.25 & 1.11 & 1.22 & 2.00 \\
\hline
\end{tabular}

a The GIF and PNG results are obtained by compressing the corresponding color image instead of the binary layers. 


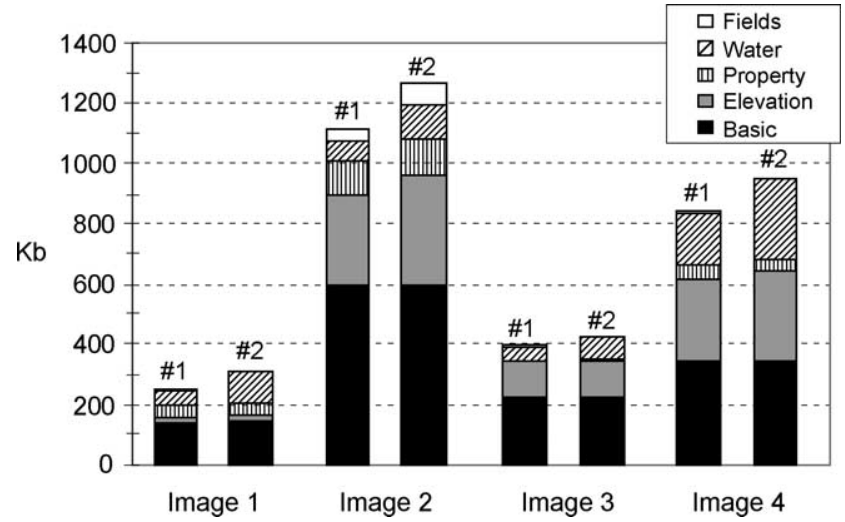

Fig. 12. The proportion of the layers in the compressed MISS files for the Set \#1 (left columns), and Set \#2 (right columns).

terms of compression performance as the model can be best optimized for images of this size. With the chosen $100 \times 100$ the files sizes are slightly bigger but more dense tiling allows more accurate buffering with less memory resources, and smaller transmission and decompression delays.

\subsection{Retrieval timings}

We consider next the retrieval performance of the proposed system by measuring time required to transmit and decompress a desired part of the map. We assume that the client device buffers the image data according to the nearest block boundaries. In other words, the client devices stores in the memory the pixels of the current view, plus additional outside pixels from the blocks across the screen boundary. In this way, we need to transmit and decompress, on average, the exact amount of pixels required to update the view.

The decompression times are summarized in Table 4 when decompressing the complete $5000 \times 5000$ images. The results show that the better compression performance of the MISS has been obtained at the cost of 10-20 times slower decoding speed in comparison to GIF and PNG. The decoding speed of MISS corresponds to 973,710 pixels

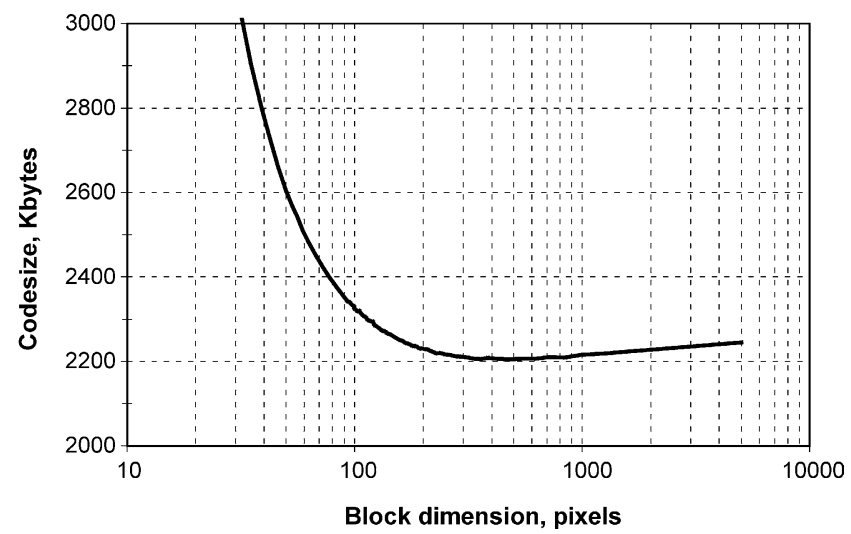

Fig. 13. The effect of the block size on the code size for the Set \#1.
Table 4

Decompression times (in seconds) for the Set \#1 using a processor of 1000 MIPS

\begin{tabular}{llll}
\hline Image & \multicolumn{2}{l}{ Method } & PNG \\
\cline { 2 - 4 } & MISS & GIF & 1.2 \\
\hline Image 1 & 25.9 & 2.1 & 1.4 \\
Image 2 & 31.4 & 2.5 & 1.1 \\
Image 3 & 18.4 & 2.2 & 1.3 \\
Image 4 & 27 & 2.2 & 5.0 \\
Total & 102.7 & 9.0 & \\
\hline
\end{tabular}

per seconds for the images in the Set \#1, on average. Using this result, we then calculated the sample retrieval timings for several different screen sizes with varying computing power and transmission speeds.

We consider the following three transmission networks:

- GSM, capable of 9600 bits per second,

- high speed GSM, capable of 14,400 bits per second,

- GRPS network, capable of $48 \mathrm{~kb} / \mathrm{s}$.

Typical hand-held devices have relatively low computing power with only a few MIPS but the computing power of these devices is developing rapidly. We consider three speeds $(10,50,100$ MIPS) that roughly correspond to the computing power of the current and forth-coming low-cost compact devices. The retrieval timings can be calculated as follows:

Transmission time

$=$ compressed data size/channel bandwidth

Decompression time

$=$ uncompressed image size/decoding speed

The results are summarized in Table 5 in case of retrieving data for a full screen. The results show that the data for a reasonable size screen can be transmitted and decompressed in real-time using existing networks and devices of reasonably low computing speed. For example,

Table 5

Transmission and decompression times (in seconds) for retrieving a full screen

\begin{tabular}{|c|c|c|c|c|c|c|}
\hline \multirow[t]{2}{*}{ Screen size } & \multicolumn{3}{|c|}{ Transmission times } & \multicolumn{3}{|c|}{ Decompression times } \\
\hline & $\begin{array}{l}9600 \text { bps } \\
\text { (GSM) }\end{array}$ & $\begin{array}{l}\text { 14,400 bps } \\
\text { (hs-GSM) }\end{array}$ & $\begin{array}{l}48 \mathrm{kbps} \\
\text { (GRPS) }\end{array}$ & $\begin{array}{l}10 \\
\text { MIPS }\end{array}$ & $\begin{array}{l}50 \\
\text { MIPS }\end{array}$ & $\begin{array}{l}100 \\
\text { MIPS }\end{array}$ \\
\hline $100 \times 100$ & 0.22 & 0.15 & 0.04 & 1.03 & 0.21 & 0.10 \\
\hline $150 \times 150$ & 0.49 & 0.33 & 0.10 & 2.31 & 0.46 & 0.23 \\
\hline $200 \times 200$ & 0.88 & 0.58 & 0.17 & 4.11 & 0.82 & 0.41 \\
\hline $250 \times 250$ & 1.37 & 0.91 & 0.27 & 6.42 & 1.28 & 0.64 \\
\hline
\end{tabular}


Table 6

Amount of work (relative to update of full screen) when refreshing a view after a diagonal scroll of 100 pixels in each dimension (corresponds to one block)

\begin{tabular}{llcc}
\hline Screen size & $\begin{array}{l}\text { Block size } \\
50 \times 50\end{array}$ & $100 \times 100$ & $200 \times 200$ \\
\hline $100 \times 100$ & $75 \%$ & $100 \%$ & $400 \%$ \\
$150 \times 150$ & $56 \%$ & $89 \%$ & $178 \%$ \\
$200 \times 200$ & $44 \%$ & $75 \%$ & $100 \%$ \\
$250 \times 250$ & $36 \%$ & $64 \%$ & $96 \%$ \\
\hline
\end{tabular}

the screen size of $150 \times 150$ pixels equals to 22,500 pixels, and $0.21 \cdot 22,500=4725$ bits in the compressed format. Let us then consider the GSM channel and a lowperformance client device with 50 MIPS. In this case, the transmission of a full screen takes about $0.33 \mathrm{~s}$ and the decompression about $0.57 \mathrm{~s}$. The timings vary depending on the complexity of the blocks; the most complex image regions take about twice as that of the average case.

In the case of movement, the view is scrolled and a new portion of data is retrieved. We consider a diagonal movement by amount of one block $(b \times b$ pixels $)$. The proportion of data to be retrieved is $\left(x b+y b-b^{2}\right)$ pixels, where $x \times y$ is the size of the screen, and $b \times b$ the size of a block. The proportion of data to be retrieved is summarized in Table 6 using various block sizes. Using the default block size of $100 \times 100$ pixels, we must decompress $64-100 \%$ of the data shown on screen size. Smaller block sizes would improve the situation but also increase the file sizes, and therefore, increase the transmission delays.

\subsection{Memory requirements}

We consider next the memory requirement of the client device. Memory is needed both for the decompression software, and for storing the uncompressed image blocks. The decompression software can be fitted into $64 \mathrm{~kb}$ of object code (standard libraries not included), or in a $100 \mathrm{~kb}$ Windows DLL (everything included). This is hardly a bottleneck. Besides the Codeq, we need memory also for buffering the image data. The following parameters must be set:

- $L=$ number of layers per image

- $K=$ number of images stored

- $C=$ number of contexts (1024 by default).

Let us suppose that we store $K$ images of different scales in the memory, and each of the images have $L$ layers. We can safely assume (on the basis of the discussion in Section 2.3) that the number of layers is never more than eight. Thus, we can store the layers one pixel per byte (one bit per layer). Furthermore, we assume that the chosen block size is within the range $50-100 \%$ of the screen size in both
Table 7

Memory requirements (in bytes) of the data buffering in the client device, and the sample numbers for the parameters: $B=2500, L=5, K=4, C=$ $1024, b=100$

\begin{tabular}{|c|c|c|c|c|}
\hline & Per block & Per layer & Per image & Total \\
\hline \multicolumn{5}{|l|}{ Client device } \\
\hline I/O data buffers & - & - & - & $2 \cdot b^{2}$ \\
\hline Image header & - & - & 400 & $L \cdot 400$ \\
\hline Coding parameters & - & 616 & $L \cdot 616$ & $K L \cdot 616$ \\
\hline Statistical model & - & $56+C \cdot 3$ & $L \cdot 56+L C \cdot 3$ & $K L \cdot 56+K L C \cdot 3$ \\
\hline Image data & - & $N / 8$ & $N$ & $K N$ \\
\hline \multicolumn{5}{|l|}{ Sample numbers } \\
\hline I/O data buffers & - & - & - & 20,000 \\
\hline Image header & - & - & 400 & 2000 \\
\hline Coding parameters & - & 616 & 3080 & 12,320 \\
\hline Statistical model & - & 3128 & 15,640 & 64,560 \\
\hline Image data & - & - & 90,000 & 360,000 \\
\hline
\end{tabular}

directions. For example, the block size $100 \times 100$ fulfill this requirement for screen sizes up to $200 \times 200$ pixels. The main consequence of this assumption is that we need to buffer only $3 \times 3$ image blocks to form a bounding box around the current view. Using the default 10-pixel context template, the number of contexts is $2^{10}=1024$.

The memory requirements are summarized in Table 7 . First, we need I/O data buffers for a single block; one buffer for the input (compressed) data and another one for the output (uncompressed) data. Image header then takes 400 bytes per image, and the coding parameters 616 bytes per layer. For each layer, we must also store the model depending on the choice of static or forward-adaptive approach. The model data takes 3 bytes per context plus 56 bytes overhead. Assuming that we have the block size of $100 \times 100$ pixels, $L=5$ layers per image, and $K=4$ images stored (e.g. images with the scale $1: 20,000,1: 40,000,1: 100,000,1: 250,000)$, the overhead of these parts sum up to about $100 \mathrm{~kb}$. The data of one images takes $9 \cdot 100^{2}=90,000$ bytes, which sums up to 360,000 bytes in total.

\section{Conclusions}

We have proposed a MISS for real-time applications that use portable devices with low memory and computing resources. The system architecture is designed to minimize storage size, transmission time, and memory requirements of the user device. Compact image size is achieved by dividing the image into semantic binary layers, which are then compressed using the state-of-art context-based method. Direct access to the compressed image file allows to transmit/decompress only the necessary part of the image needed. This minimizes the transmission time and memory requirement in the user device are minimized.

Mobile map imaging systems can be designed on the basis of the proposed methodology. It remains to be seen when such dynamic map imaging systems will become 
available for broad use-technically there should be no restrictions anymore.

\section{Acknowledgements}

The work was supported by the National Technology Agency of Finland (TEKES) as the projects. Real-time cartography imaging and dynamic use of maps in mobile environment.

\section{References}

[1] M.-J. Kraak, et al., Cartography: Visualization of Spatial Data, Addison-Wesley, Reading, MA, 1996.

[2] M.-J. Kraak, A. Brown, Web Cartography, Taylor \& Francis, London, 2000.

[3] E.D. Kaplan (Eds.), Understanding GPS: Principles and Applications, Artech House Telecommunications Library, 1996, March.

[4] S. Dye, S. Buckingham, Mobile Positioning, Mobile Lifestreams (1999).

[5] E.A. Fox, et al. (Eds.), Digital Libraries, [Special Issue of] Communications of the ACM, 38(4), 1995.

[6] H. Samet, Applications of Spatial Data Structures: Computer Graphics, Image Processing, GIS, Addison-Wesley, Reading, MA, 1989.

[7] GeoData, CD-tiekartasto Suomi 1:250,000 (CD Roadmap Catalog Finland), WSOY, Helsinki, 1999, (in Finnish).

[8] H.W. Gellersen, P.J. Thomas (Eds.), Proceedings of the Second International Symposium on Handheld and Ubiquitous Computing (HUC'2000), Bristol, UK, September 25-27, Springer, Berlin, 2000.

[9] R.B. Arps, T.K. Truong, Comparison of international standards for lossless still image compression, Proceedings of the IEEE 82 (6) (1994) 889-899. June.

[10] J.D. Murray, W. vanRyper, D. Russell (Eds.), Encyclopedia of Graphics File Formats, second ed., O'Reilly Associates, Inc, Cambridge, MA, 1996.

[11] B.G. Haskell, et al., Image and video coding-emerging standards and beyond, IEEE Transactions on Circuits and Systems for Video Technology 8 (7) (1998) 814-837.

[12] ISO/IEC, Progressive Bi-level Image Compression, 11544, ITU-T Recommendation T.82, 1993.

[13] ISO/IEC, Final committee draft for ISO/IEC International Standard, 14492, 1999 (http://www.jpeg.org/public/jbigpt2.htm).

[14] P. Howard, F. Kossentini, B. Martins, S. Forchammer, W.J. Rucklidge, The emerging JBIG2 standard, IEEE Transactions on Circuits and Systems for Video Technology 8 (7) (1998) 838-848.

[15] J. Miano, Compressed Image File Formats: JPEG, PNG, GIF, XBM, BMP, (ACM Press), Addison-Wesley, Boston, MA, 1999.
[16] G. Roelofs, PNG: The Definitive Guide, O'Reilly and Associates, Cambridge, MA, 1999, June.

[17] W.B. Pennebaker, J.L. Mitchell, JPEG Still Image Data Compression Standard, Van Nostrand Reinhold, New York, 1993.

[18] G.G. Langdon, J. Rissanen, Compression of black-white images with arithmetic coding, IEEE Transactions on Communications 29 (6) (1981) 858-867. June.

[19] W.B. Pennebaker, J.L. Mitchell, G.G. Langdon, R.B. Arps, An overview of the basic principles of the Q-coder adaptive binary arithmetic coder, IBM Journal of Research and Development 32 (6) (1988) 717-726.

[20] P. Fränti, E. Ageenko, P. Kopylov, S. Gröhn, Compressing Multicomponent Digital Maps Using JBIG2, IEEE International Conference on Acoustics, Speech, and Signal Processing (ICASSP'02), Orlando, Florida, USA, May, vol. 3, 2002, pp. 2677-2680.

[21] P. Kopylov, P. Fränti, Compression of map images by multi-layer context tree modeling, IEEE Transactions on Image Processing (2004) (in press).

[22] D. Tompkins, F. Kossentini, Additional Extension Segments for JBIG2, ISO/IEC JTC 1/SC 29/WG1 (ITU-T SG8), Document No. 1318, 1999, July.

[23] M.J. Weinberger, J. Rissanen, R. Arps, Application of universal context modeling to lossless compression of gray-scale images, IEEE Transactions on Image Processing 5 (4) (1996) 575-586. April.

[24] E.I. Ageenko, P. Fränti, Enhanced JBIG-based compression for satisfying objectives of engineering document management system, Optical Engineering 37 (5) (1998) 1530-1538. May.

[25] E.I. Ageenko, P. Fränti, Compression of large binary images in digital spatial libraries, Computer and Graphics 24 (1) (2000) 91-98.

[26] E.I. Ageenko, P. Fränti, Forward-adaptive method for compressing large binary images, Software Practice and Experience 29 (11) (2000) 943-952.

[27] E.I. Ageenko, P. Kopylov, P. Fränti, On the Size and Shape of Multilevel Context Templates for Compression of Map Images, IEEE International Conference on Image Processing (ICIP'01), Thessaloniki, Greece, October, vol. 3, 2001, pp. 458-461.

[28] P. Fränti, P. Kopylov, V. Veis, Dynamic Use of Map Images in Mobile Environment, IEEE International Conference on Image Processing (ICIP'02), Rochester, New York, USA, September, vol. 3, 2002, pp. 917-920.

[29] P. Fränti, Dynamic map handling, GIM International 17 (1) (2003) 28-31. January.

[30] T. Welch, A technique for high-performance data compression, IEEE Computer 17 (6) (1984) 8-19. June.

[31] J. Ziv, A. Lempel, Compression of individual sequences via variablerate coding, IEEE Transactions on Information Theory 24 (1978) $530-536$.

[32] J. Ziv, A. Lempel, A universal algorithm for sequential data compression, IEEE Transactions on Information Theory 23 (1977) $337-343$.

[33] National Land Survey of Finland, Opastinsilta 12 C, P.O. Box 84, 00521 Helsinki, Finland (http://www.nls.fi/index_e.html) 